\title{
A METHOD OF DERIVING SOLUTIONS OF A CLASS OF BOUNDARY VALUE PROBLEMS
}

\author{
by F. J. LOCKETT \\ (Received 22nd November 1958)
}

\section{Introduction}

In many branches of applied mathematics there exists a class of problems which depend for their solution upon the integration of a set of simultaneous linear partial differential equations subject to certain boundary conditions. In all but the simplest cases it is not practicable to deal with these equations by standard methods. For problems involving infinite regions, solutions can often be found by the use of integral transforms. However, in many problems we are concerned with media of finite extent, so that if we are to make a direct application of this method, we shall have to use finite transforms, and under certain conditions these are much more difficult to apply than transforms over an infinite range.

In this paper we shall show how, by considering a modified problem in the entire (infinite) space, we can use transforms over the infinite range to obtain the solutions of the problem for the finite medium.

\section{Illustrative Example}

Before attempting to state the principles of the method in general terms we shall use the method to solve a relatively simple problem. It should then be easier for the reader to understand the general formulation, by comparing it with this solution.

Temperature distribution in an infinite cylinder due to a surface temperature $\theta_{0}(z, t)$.

As our example we shall find the temperature distribution in an infinitely long circular cylinder due to the application of a boundary temperature $\theta_{0}(z, t)$ on the surface $r=a$. Thus the problem is mathematically equivalent to finding a solution, in the region $r \leqslant a$, of the heat conduction equation

$$
\frac{\partial^{2} \theta}{\partial r^{2}}+\frac{1}{r} \frac{\partial \theta}{\partial r}+\frac{\partial^{2} \theta}{\partial z^{2}}=\kappa \frac{\partial \theta}{\partial t}
$$

subject to the boundary condition that $\theta=\theta_{0}(z, t)$ on $r=a$.

Instead of solving this problem directly, we shall consider a modified problem in an infinite medium. We shall find the effect of a (for the moment unknown) heat source $\Theta(z, t)$ which is concentrated on the radius $r=a$. We shall then choose $\Theta(z, t)$ in such a way that $\theta=\theta_{0}(z, t)$ on $r=a$. Our solution will therefore satisfy

$$
\frac{\partial^{2} \theta}{\partial r^{2}}+\frac{1}{r} \frac{\partial \theta}{\partial r}+\frac{\partial^{2} \theta}{\partial z^{2}}=\kappa \frac{\partial \theta}{\partial t}-\Theta(z, t) \delta(r-a)
$$

subject to the condition that $\theta=\theta_{0}(z, t)$ on $r=a$.

Now, within the region $r<a$, equations (1) and (2) are identical, so that, 
within this region, our solutions for the modified problem are also the solutions required for the cylinder problem.

If we define the transforms

$$
\begin{aligned}
& \bar{\theta}(\xi, \zeta, \omega)=\frac{1}{2 \pi} \int_{-\infty}^{\infty} \int_{-\infty}^{\infty} e^{i(\zeta z+\omega t)} d z d t \int_{0}^{\infty} r \theta(r, z, t) J_{0}(\xi r) d r \\
& f^{0}(\zeta, \omega)=\frac{1}{2 \pi} \int_{-\infty}^{\infty} \int_{-\infty}^{\infty} f(z, t) e^{i(\zeta z+\omega t)} d z d t
\end{aligned}
$$

equation (2) transforms to

$$
\left(\xi^{2}+\zeta^{2}-i \omega \kappa\right) \bar{\theta}=a J_{0}(\xi a) \Theta 0
$$

so that

$$
\begin{aligned}
& \theta^{0}(r, \zeta, \omega)=\frac{1}{2 \pi} \int_{-\infty}^{\infty} \int_{-\infty}^{\infty} \theta(r, z, t) e^{i(\zeta z+\omega t)} d z d t \\
& =\int_{0}^{\infty} \xi J_{0}(\xi r) \bar{\theta}(\xi, \zeta, \omega) d \xi \\
& =a \Theta^{0} \int_{0}^{\infty} \xi\left(\xi^{2}+\zeta^{2}-i \omega \kappa\right)^{-1} J_{0}(\xi a) J_{0}(\xi r) d \xi \\
& =a \Theta^{0} I_{0}(k r) K_{0}(k a) \text { for } r \leqslant a
\end{aligned}
$$

where $k^{2}=\zeta^{2}-i \omega k$ and we choose for $k$ the branch with the positive real part.

We can now apply the transformed boundary condition $\theta^{0}(a, \zeta, \omega)=\theta_{\mathbf{0}}^{0}(\zeta, \omega)$ to get

$$
a \Theta^{0}=\theta_{0}^{\circ} / I_{0}(k a) K_{0}(k a)
$$

and substitution of this expression into (7) gives

$$
\theta^{0}(r, \zeta, \omega)=\theta_{0}^{0} I_{0}(k r) / I_{0}(k a)
$$

Finally we use (8) and the transforms inverse to (6) to give us the temperature distribution

$$
\theta(r, z, t)=\frac{1}{2 \pi} \int_{-\infty}^{\infty} \int_{-\infty}^{\infty} \theta_{0}^{0}(\zeta, \omega) \frac{I_{0}\left\{r\left(\zeta^{2}-i \omega \kappa\right)^{1}\right\}}{I_{0}\left\{a\left(\zeta^{2}-i \omega \kappa\right)^{1}\right\}} e^{-i(\zeta z+\omega t)} d \zeta d \omega
$$

where we take the square root with the positive real part. It is easily verified that this expression satisfies equation (1) and the boundary condition $\theta=\theta_{0}(z . t)$ on $r=a$.

\section{General Physical Description of Method}

Suppose that the medium in our problem occupies the region $D$ and is acted upon by various "causes" which we shall denote by $C$. These causes may be body forces, heat sources, electric or magnetic fields, sources, sinks, etc. On the boundaries $S_{1}, S_{2}, \ldots$ of $D$ there are a set of boundary conditions which we shall denote by $T$. The set of equations $T$ may express the fact that stress components, temperature, temperature gradient, potential, etc., are known on these boundaries. Suppose that our physical knowledge of the problem allows us to formulate it as a simultaneous set of linear partial 
differential equations $E$, which we have to solve within the region $D$ in accordance with the boundary conditions $T$.

We consider now a different problem. Let the medium occupy the entire space $X$, which may be of one, two or three dimensions according to the problem in question. In addition to the causes $C$ acting within the region $D$, we now consider a further system of causes $C^{*}$ acting on the surfaces $S_{1}, S_{2}, \ldots$ For each boundary $C^{*}$ contains a complete set of causes concentrated on this boundary. By a "complete" set we mean that it should contain all the types of cause relevant to the particular branch of the subject. Thus, in an elastic problem, it should contain body forces in all possible directions, whilst in a thermoelastic problem it should also contain a concentrated heat source. This ensures that there are as many members in the $C^{*}$ as there are boundary conditions in the original problem.

Suppose that the physics of the modified problem leads to a set of governing equations $E^{*}$. Within the interior of the region $D$ the sets of equations $E^{*}$ and $E$ are identical since the $C^{*}$ are zero within this region. We now use the transform method to obtain solutions of the equations $E^{*}$ within the entire space $X$. These solutions will depend on the $C^{*}$, which up to now are unknown functions. We now choose expressions for them so that the conditions $T$ are satisfied.

Our solutions therefore satisfy the boundary conditions, and within $D$ they are solutions of $E$. Thus we have found solutions to our original problem. As far as the finite medium problem is concerned the solutions have no meaning whatsoever outside the region $D$.

\section{Restrictions on Type of Problem Considered}

The method is only applicable to a certain class of problems. The restrictions which must be placed on the type of problem considered are mainly concerned with the type of boundary and the type of boundary condition which are permitted. In fact, we only require that the governing equations should be a set of linear partial differential equations which can be reduced to a set of algebraic equations by the application of a suitable multiple integral transform over the entire space. We place no restriction either on the number of independent variables or on the number of unknowns (as long as this is equal to the number of equations!)

The boundary conditions may be given by specifying the boundary values of functions of the unknowns and/or their derivatives, but each such specification must be made over the whole of that boundary. Thus it is not permissible to have mixed boundary conditions on any boundary, although the conditions on one boundary may be entirely different from those on another boundary.

The other restriction is the form which the boundary itself can take. Each boundary must be of the form; some coordinate $x_{1}=$ constant, and in any one problem all the boundaries must be given by putting the same coordinate equal to different constant values. Thus it is not permissible to consider the 
quarter plane bounded by $x=0(y>0)$ and $y=0(x>0)$, though it is possible to consider this domain if we use polar coordinates, since the boundaries are then $\theta=0$ and $\theta=\pi / 2$. This last condition does of course, restrict the number of problems which can be tackled by the method. However, we can see that by using suitable coordinates we can tackle problems involving the semiinfinite plane, infinite strip, semi-infinite space, infinite plate, interior and exterior of circle, circular ring, infinite sector, infinite circular cylinder and tube, infinite space with cylindrical cavity, infinite wedge, sphere, shell, infinite cone, etc. Thus an interesting class of problems still remains.

\section{General Mathematical Formulation}

In Section 3 we described the method in physical terms. It is, of course, possible to give a purely mathematical formulation, and this is the purpose of the present section.

Suppose that we have a set of linear partial differential equations

$$
\mathbf{L y}-\mathbf{f}=\mathbf{0}
$$

where $\mathbf{L}$ is an $n \times n$ matrix of linear partial differential operators in the independent variables $x_{1}, x_{2}, \ldots x_{r}, \mathrm{y}$ is the column matrix $\left\{y_{1} y_{2} \ldots y_{n}\right\}$ of the unknown functions $y_{i}=y_{i}\left(x_{1}, x_{2} \ldots x_{r}\right)$ and $\mathbf{f}$ is the column matrix $\left\{f_{1} f_{2} \ldots f_{n}\right\}$ of the known functions $f_{i}=f_{i}\left(x_{1}, x_{2}, \ldots x_{r}\right)$. We wish to find solutions of these equations within the domain $a_{1} \leqslant x_{1} \leqslant a_{2}$, all $x_{2} \ldots x_{r}$ (time may be included in the independent variables) subject to certain boundary conditions on $x_{1}=a_{1}$ and $x_{1}=a_{2}$. These conditions may include functions differentiated with respect to any of the variables.

Consider now the set of equations

$$
\mathbf{L y}-\mathbf{f}=\boldsymbol{\phi}
$$

where $\phi$ is a column matrix whose elements are of the form

$$
\phi_{i}=g_{1 i}\left(x_{2}, \ldots x_{r}\right) \delta\left(x_{1}-a_{1}\right)+g_{2 i}\left(x_{2}, \ldots x_{r}\right) \delta\left(x_{1}-a_{2}\right)
$$

so that $\phi_{i}=0$ for $a_{1}<x_{1}<a_{2}$.

Equation (12) can be written in matrix form as

$$
\phi=\mathbf{g}_{1} \delta\left(x_{1}-a_{1}\right)+\mathbf{g}_{2} \delta\left(x_{1}-a_{2}\right) .
$$

The solution of the problem can now be completed in four steps.

(i) Transform equations (11) over the entire range of $x_{1}, x_{2} \ldots x_{r}$ so that

$$
\overline{\mathbf{L y}}-\overline{\mathbf{f}}=\bar{\phi} \text {. }
$$

Suppose that

$$
\begin{aligned}
\overline{\mathbf{L y}} & =\mathbf{m}\left(\xi_{1}, \ldots \xi_{r}\right) \mathbf{y} \\
\bar{\phi} & =\mathbf{g}_{1}^{0}\left(\xi_{2}, \ldots \xi_{r}\right) h_{1}\left(\xi_{1}, a_{1}\right)+\mathbf{g}_{2}^{0}\left(\xi_{2}, \ldots \xi_{r}\right) h_{2}\left(\xi_{1}, a_{2}\right)
\end{aligned}
$$

where the superscript " 0 " denotes the transform with respect to all 
co-ordinates except $x_{1}$, and $h_{1}$ and $h_{2}$ are the transforms of the delta functions with respect to $x_{1}$. Equation (14) then gives

$$
\left.\overline{\mathbf{y}}=\mathbf{m}^{-1} \overline{\{}+\mathbf{g}_{1}^{0} h_{1}+\mathrm{g}_{2}^{0} h_{2}\right\}
$$

(ii) Denote by $\mathscr{T}^{-1}$ the inverse transform with respect to $\xi_{1}$. Then (15) gives

$$
\mathscr{T}^{-1} \overline{\mathbf{y}}=\mathbf{y}^{\mathbf{0}}=\mathscr{T}^{-1}\left\{\mathbf{m}^{-1} \mathbf{\mathbf { f }}\right\}+\mathscr{T}^{-1}\left\{\mathbf{m}^{-1} h_{\mathbf{1}}\right\} \mathbf{g}_{1}^{0}+\mathscr{T}^{-1}\left\{\mathbf{m}^{-1} h_{2}\right\} \mathbf{g}_{2}^{0}
$$

since $\mathbf{g}_{1}^{0}$ and $\mathbf{g}_{2}^{0}$ are not functions of $\xi_{1}$. When these transforms are evaluated (16) can be written in the form

$$
\begin{aligned}
\mathbf{y}^{\mathbf{0}}\left(x_{1}, \xi_{2}, \ldots \xi_{r}\right) & =\mathbf{u}\left(x_{1}, \xi_{2}, \ldots \xi_{r}\right) \\
& +\mathbf{v}\left(x_{1}, \xi_{2} \ldots \xi_{r}\right) \mathbf{g}_{1}^{0}\left(\xi_{2}, \ldots \xi_{r}\right)+\mathbf{w}\left(x_{1}, \xi_{2} \ldots \xi_{r}\right) \mathbf{g}_{2}^{0}\left(\xi_{2} \ldots \xi_{r}\right)
\end{aligned}
$$

(iii) Apply the transformed boundary conditions to (17) and solve the resulting equations for $\mathbf{g}_{1}^{0}$ and $\mathbf{g}_{2}^{0}$. Substitute these values into (17) to obtain an expression for $\mathrm{y}^{\mathbf{0}}$.

(iv) Apply the inverse transform to $\mathrm{y}^{0}\left(x_{1}, \xi_{2}, \ldots \xi_{r}\right)$ and obtain an expression for $\mathrm{y}\left(x_{1}, \ldots x_{r}\right)$.

Within the region $a_{1}<x_{1}<a_{2}$ these solutions satisfy (10) since $\phi=0$ there, and they also satisfy the boundary conditions on $x_{1}=a_{1}$ and $x_{1}=a_{2}$. They are therefore the required solutions.

\section{Discussion}

The simple example given in $\S 2$ is easily solved by the standard method. In fact, it is only necessary to transform (1) with respect to $z$ and $t$ to obtain a differential equation whose solution is easily seen to be of the form (8). Thus we should give some justification for producing a method which at first sight seems to be more complicated than the standard ones.

Theoretically all problems of the type envisaged here can be solved by standard methods, and it is best that simple problems should be solved by this method. However, for more complicated problems involving several unknown functions the standard procedure becomes very difficult to apply. As an example, let us consider the amount of work necessitated by the standard method and by the present method for the following problem.

We require the solutions to a system of $n$ simultaneous $m$ 'th order linear partial differential equations in $n$ unknowns and $r$ independent variables.

(i) Standard method : Transform each equation by an $(r-1)$ dimensional transform, giving $n$ simultaneous $m^{3}$ th order differential equations. Eliminate the unknowns to give $n(m n)^{\text {th }}$ order differential equations. Solve these equations and apply the $(r-1)$ dimensional inverse transforms.

(ii) Present method : Transform each equation by an $r$ dimensional transform, giving $n$ simultaneous algebraic equations. Solve this set of equations and apply the $r$ dimensional inverse transforms.

Thus, at the expense of a one-dimensional transform on each equation and a one-dimensional inverse transform in each solution, we gain the advantage

E.M.S. - $-\mathbf{K}$ 
of having to solve a set of algebraic equations rather than a set of differential equations which are not easy to obtain. Even for fairly small values of $m$, $n$ and $r$ this advantage, and the fact that the present method is more systematic, greatly outweigh the disadvantages. As an example the reader is referred to an application of this method by Lockett, 1959, which appears as a companion paper in this issue. In particular the reader is referred to equations (28), which, apart from the delta functions, are the equations whose solutions are required.

Nothing has been said so far about the values which should be taken for the unknown functions at the limits of integration, when transforming the differential equations. In many problems the values at one limit will be given by the corresponding finite medium problem, whilst the values at the other limit will be arbitrary. For example, in the example of $\S 2$ the values at $r=0$ are given, but it can easily be verified that the result (9) is independent of the conditions introduced at $r=\infty$. This is to be expected from physical reasoning.

The method described in $\S 3$ and $\S 5$ also suggests the possibility of extension to the case of concentrating the causes $C^{*}$ outside the boundary on the surface $x_{1}=d$. However, we must then be more careful in order to retain mathematical rigour of the analysis. For instance, in the example of $\S 2$ we could have chosen a heat source on the radius $r=d$, where $d>a$. The analysis then continues as before and leads to the result (9). However, it can be seen that the expression for $\Theta^{0}$ is now

$$
d \Theta^{0}=\frac{\theta_{0}^{0}}{I_{0}(k a) K_{0}(k d)}+\frac{A^{0}(\omega, \zeta)}{K_{0}(k d)}
$$

where $A^{0}$ is the transform of a function of the conditions introduced at $r=\infty$. If $A=0$, this will lead to an integral expression for $\Theta$ which, for many choices of $\theta_{0}$ will be divergent. When $d=a$ this mathematical difficulty disappears. Otherwise it may be possible to choose conditions at $r=\infty$ in such a way that the expression (18) converges.

In the example given in the paper referred to above, the causes $C^{*}$ have been placed on the radius $r=d$. Mathematical rigour can be attained either by taking $d=a$, or by introducing suitable conditions at the limits of integration, though it will be seen that this does not make any difference to the final results.

\section{ACKNOWLEDGEMENTS}

I am indebted to $\mathrm{Mr} \mathrm{B}$. Noble for his constructive criticism of the original draft of this paper, and to the Department of Scientific and Industrial Research for the award of a Research Studentship during the period in which the work was done.

\section{REFERENCE}

F. J. Lockett, Proc. Edin. Math. Soc., 11 (1959), 153-159.

\section{Departaent of Mathematics \\ UNIVERSITY OF GLASGOW}

